\title{
Jostent covered stent placement for emergency reconstruction of a ruptured internal carotid artery during or after transsphenoidal surgery
}

\author{
Byung Moon Kim, MD, PhD, ${ }^{1}$ Pyoung Jeon, MD, ${ }^{2}$ Dong Joon Kim, MD, ${ }^{1}$ Dong Ik Kim, MD, ${ }^{1}$ \\ Sang Hyun Suh, MD, ${ }^{3}$ and Keun Young Park, MD ${ }^{4}$
}

Departments of ${ }^{1}$ Radiology and ${ }^{4}$ Neurosurgery, Severance Hospital, Yonsei University College of Medicine; ${ }^{2}$ Department of Radiology, Samsung Medical Center, Sungkyunkwan University School of Medicine; and 'Department of Radiology, Gangnam Severance Hospital, Yonsei University College of Medicine, Seoul, South Korea

\begin{abstract}
OBJECT Internal carotid artery (ICA) rupture during transsphenoidal surgery (TSS) is an extremely difficult complication to treat. This study aimed to evaluate the immediate and long-term outcomes of covered stent placement for emergency reconstruction of ruptured ICAs during or after TSS.

METHODS Seven patients underwent covered stent placement for emergency reconstruction of a ruptured ICA during or after TSS. The safety and effectiveness of covered stent placement for emergency reconstruction of ruptured ICAs were retrospectively analyzed.
\end{abstract}

RESULTS Pretreatment angiography showed active bleeding in 6 patients ( 5 intraoperative and 1 postoperative) and a pseudoaneurysm in 1 patient. Of the 6 patients with active bleeding, 5 were treated with a successive operation to control active bleeding. The other patient was treated just after cardiopulmonary resuscitation due to massive nasal bleeding 20 days after revision of TSS. All active bleeding was controlled immediately after covered stent insertion in these 6 patients. One patient showed a gap between the covered stent and ICA wall without active bleeding 30 minutes after glycoprotein Ilb/Illa inhibitor administration due to in-stent thrombosis. The gap was occluded with coil embolization after completion of the temporarily suspended TSS. The seventh patient, whose ICA tear was treated with surgical suture, underwent covered stent placement for a pseudoaneurysm detected on postoperative Day 2. During a mean follow-up period of 46 months (range 12-85 months), all patients had excellent outcomes (modified Rankin Scale score of 0). All the stented ICAs were patent on vascular imaging follow-up at a mean of 34 months (range 12-85 months).

CONCLUSIONS Covered stents appear to be a safe and effective option for emergency reconstruction of ruptured ICAs during or after TSS.

http://thejns.org/doi/abs/10.3171/2014.10.JNS14328

KEY WORDS covered stent; internal carotid artery; transsphenoidal surgery; vascular disorders

A LTHOUGH its incidence is believed to be low, intraoperative bleeding due to direct injury to the internal carotid artery (ICA) may be one of the most difficult complications to address during transsphenoidal surgery (TSS).$^{13}$ This complication requires prompt recognition and management to prevent potentially life-threatening consequences or severe neurological sequelae. Direct surgical repair with suture or clip graft is difficult due to limited exposure. Surgical packing can be used to instantly control active intraoperative bleeding, which might be ineffective in addition to being associated with a significant rate of neurological complications.,.$^{2,13}$ Endovascular parent artery occlusion is an effective alternative to surgical means $;{ }^{2}$ however, parent artery sacrifice requires sufficient cross-collaterals via communicating arteries; otherwise bypass surgery should be performed first. Nevertheless, it is actually impossible to perform the carotid occlusion test for evaluation of cross-collaterals in the emergency setting. Furthermore, emergency bypass surgery is also difficult and technically very demanding. Covered stents may be an alternative option to parent artery trapping in such emergency settings, but only a few cases reported reconstruction

ABBREVIATIONS CCF = carotid-cavernous fistula; ICA = internal carotid artery; MCA = middle cerebral artery; mRS = modified Rankin Scale; TSS = transsphenoidal surgery.

SUBMITTED February 25, 2014. ACCEPTED October 8, 2014.

INCLUDE WHEN CITING Published online November 21, 2014; DOI: 10.3171/2014.10.JNS14328.

DISCLOSURE The authors report no conflict of interest concerning the materials or methods used in this study or the findings specified in this paper. 
of the ruptured ICA related to TSS. . $^{3,4,8,10,11,14,19}$ In this study, we present our experience of emergency reconstruction of intraoperative or postoperative ruptured cavernous ICAs with a covered stent with long-term follow-up results.

\section{Methods}

The institutional review board of Severance Hospital approved this retrospective analysis and waived patient informed consent. All patients who underwent attempted covered stent insertion for reconstruction of ruptured cavernous ICAs during or after TSS between January 2007 and January 2013 were identified from a prospectively maintained neurointerventional database in 2 academic hospitals (Severance Hospital and Samsung Medical Center). One thousand two hundred thirty cases of TSS were performed during the same period.

\section{Endovascular Procedure}

An 80-cm 6-Fr or 7-Fr shuttle-guiding sheath (Cook) was placed in the distal cervical artery with a triaxial technique using a $125-\mathrm{cm} 5-\mathrm{Fr}$ angiocatheter and a 0.035 -inch wire. A 6-Fr or 7-Fr guiding catheter (Cordis) was coaxially placed through the prepositioned shuttle sheath for enough support during delivery of a covered stent. A 300$\mathrm{cm}, 0.014$-inch exchangeable microwire (Transcend, Stryk$\mathrm{er}$, or Grandslam, Asahi Intecc) was placed in the $\mathbf{M}_{2}$ or $\mathbf{M}_{3}$ portion of the middle cerebral artery (MCA) using a microcatheter. A premounted covered stent (Jostent graft, Abbott Vascular Devices) size-matched to the proximal parent artery diameter was advanced over the prepositioned exchangeable-length microwire and positioned completely covering the rupture point of the ICA. If the ICA was too tortuous to navigate, 2 types of additional technical tips were adopted. First, a 300-cm, 0.018-inch stiff microwire (SV5, Cordis) was placed distally to the ICA or $\mathrm{M}_{1}$ proximal portion of the MCA using a 0.018-inch microcatheter to provide further support for the guiding system and to straighten the tortuous cavernous ICA. Second, if covered stent navigation remained difficult even after the first technical tip was applied, the internal guiding catheter (6-Fr or 7-Fr) was advanced to the petrous ICA over a triwire system: the prepositioned 0.014 -inch exchangeable wire, the 0.018 -inch buddy wire, and the covered stent delivery pusher. Then, advancement of the covered stent was reattempted to the target position (Fig. 1). After confirmation of the covered stent position, the balloon was inflated to the nominal pressure after the 0.018 -inch buddy wire, if inserted, was removed. If a control angiogram after initial balloon inflation showed an endoleak, the balloon was repeatedly inflated to the burst pressure. After confirming apposition of the covered stent to the vessel wall without an endoleak, the balloon was removed and a 10-minute delayed control angiogram was obtained to determine whether in-stent thrombosis occurred. When in-stent thrombosis was detected, a glycoprotein IIb/IIIa inhibitor (abciximab, 5-15 mg) was administered through the guiding catheter and repeat follow-up angiograms were obtained every 10 minutes until in-stent thrombus was completely resolved.

Intravenous administration of bolus heparin was not given to any patient. Antiplatelet premedication was not

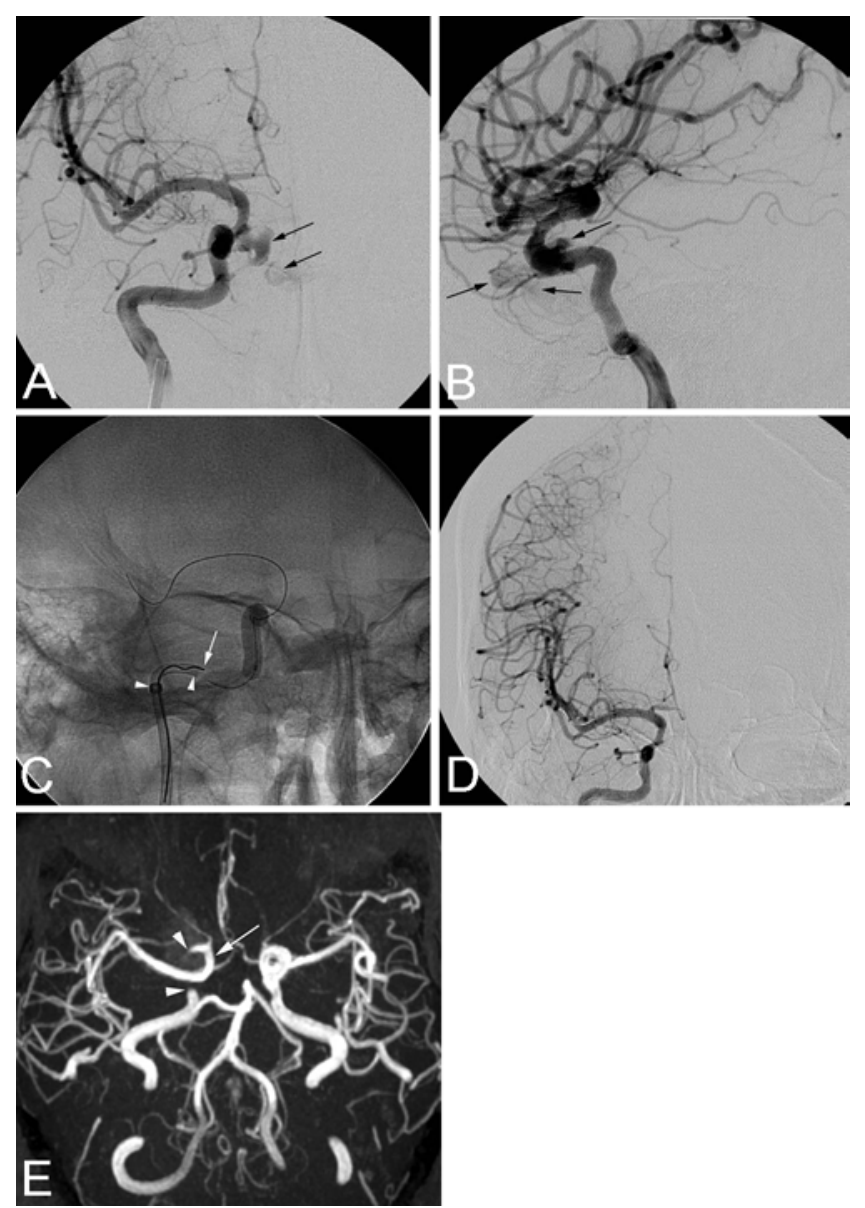

FIG. 1. Case 3. Images obtained in a 49-year-old woman with massive epistaxis 20 days after revision TSS. A and B: Frontal (A) and lateral (B) angiograms showing contrast medium leakage from the right ICA cavernous segment through the sphenoid sinus into the posterior nasal cavity (arrows). Note that the $A_{1}$ segment of the left anterior cerebral artery was hypoplastic and spastic. C: A 4/12-mm covered stent was positioned fully covering the rupture point and then deployed. Note the double guiding catheter system (arrowheads) and the 0.018-inch stiff buddy wire (arrow) for sufficient support during delivery of the covered stent. D: The 30-minute delayed control angiogram showed a patent ICA without leakage of contrast medium. E: The 13-month follow-up 3D time-of-flight MR angiogram showed a patent right ICA (arrow). The stented segment (arrowheads) of the right ICA was not seen due to metallic artifact.

given to any patient, but a loading dose of dual antiplatelet medication (aspirin $100 \mathrm{mg}$ and clopidogrel $300 \mathrm{mg}$ ) was immediately administered via nasogastric tube to all patients. Dual antiplatelet medication was maintained for at least 3-6 months and then changed to aspirin monotherapy indefinitely.

\section{Outcome Evaluation}

All electronic medical charts and radiological studies were reviewed to obtain relevant clinical and radiological data. In addition, we reviewed a prospectively maintained neurointerventional database to obtain information about the procedure itself, including adverse events. The patients' functional outcomes were evaluated according to the modified Rankin Scale (mRS) score. The functional 
outcome at the latest follow-up evaluation was defined as the final outcome. If the patients' latest follow-up evaluation was more than 3 months before the point of data collection of this study, the functional outcome was evaluated using a telephone interview.

\section{Results}

Seven patients underwent attempted covered stent insertion for reconstruction of a ruptured cavernous ICA during or after TSS. Patient demographics, type of operations, and rupture sites are detailed in Table 1. Pretreatment angiography showed active bleeding (extravasation of contrast medium) in 6 patients ( 5 intraoperative and 1 postoperative) and a pseudoaneurysm in 1 patient. Of the 6 patients with active bleeding, 5 were treated under general anesthesia in successive operations: 2 patients were treated under C-arm guidance in the operating room, and 3 patients were in the angiographic suite immediately after transfer of the patient from the operative room. The other patient was treated just after cardiopulmonary resuscitation due to hypovolemic shock secondary to massive nasal bleeding 20 days after revision of TSS. The seventh patient was initially treated with surgical suture in the operating room, but the patient showed a pseudoaneurysm at the cavernous ICA on followup angiography 2 days later and underwent covered stent placement in the same session.

Immediate and follow-up clinical and angiographic outcomes are summarized in Table 2. All attempts at covered stent insertion were completed without delivery failure. The first technical "tip" (or trick) was used in 3 cases, and the second tip was additionally needed in 2 of the 3 cases. In 6 patients with active bleeding, the bleeding was immediately controlled just after stent graft insertion. However, 1 patient showed a gap between the covered stent and ICA wall without active bleeding 30 minutes after administration of the glycoprotein IIb/IIIa inhibitor (abciximab, $15 \mathrm{mg}$ ) due to in-stent thrombosis. The patient was further treated with coil insertion into the gap 18 hours after completion of the temporarily suspended TSS as previously reported. ${ }^{15}$ In the case in which the ruptured ICA was initially treated with surgical suture, the pseudoaneurysm was excluded on the angiogram obtained just after covered stent insertion. Apart from the 1 case of in-stent thrombosis mentioned above, 2 other in-stent thromboses occurred but resolved with glycoprotein IIb/ IIIa inhibitor administration without any clinical sequelae.

During clinical follow-up of a mean duration of 46 months (range 12-85 months), all patients had excellent outcomes (mRS score of 0 ). Vascular imaging follow-up with catheter angiography $(n=5)$ or MR/CT angiography $(n=2)$ was available at least once in all 7 patients at a mean of 34 months (range 12-85 months). All the stented ICAs were patent on vascular imaging follow-up.

\section{Illustrative Cases \\ Case 3}

A 49-year-old woman developed massive epistaxis 20 days after revision of TSS due to CSF leakage. The patient suffered cardiac arrest due to hypovolemic shock 5 minutes thereafter. Emergency cardiopulmonary resuscitation with transfusion was conducted, and the patient recovered from the cardiac arrest. Emergency angiography (Fig. 1A and B) after nasal packing showed leakage of contrast medium from the right ICA cavernous segment through the sphenoid sinus into the posterior nasal cavity. Because the right anterior cerebral artery $A_{1}$ segment was hypoplastic with spasm and the right posterior communicating artery was poorly developed, covered stent insertion was attempted for emergency reconstruction of the right ICA. After placement of a double guiding catheter system and a 0.018-inch buddy wire for enough support during the covered stent navigation, a 4/12-mm covered stent (Jostent graft) was positioned to completely cover the rupture point and then deployed (Fig. 1C). Both an immediate and a 30-minute delayed control angiogram showed a patent ICA without leakage of contrast medium (Fig. 1D). The patient completely recovered, and the 13-month follow-up 3D time-of-flight MR angiogram showed a patent right ICA (Fig. 1E). The patient had no neurological symptoms at the 46-month clinical follow-up.

\section{Case 5}

A 30-year-old woman was admitted for removal of a pituitary macroadenoma encasing the left ICA cavernous segment. During removal of the macroadenoma, massive bleeding occurred due to left cavernous ICA rupture. After surgical packing, emergency C-arm angiography was performed in the operating room. The left ICA angiogram showed contrast medium extravasation from the left cavernous ICA into the sphenoid sinus (Fig. 2A). For emergency reconstruction of the left ICA, a 4/12-mm Jostent covered stent was deployed. The active bleeding immediately stopped but a small amount of contrast medium leakage was revisualized due to an endoleak on the 10-minute

TABLE 1. Summary of patient demographics, type of operations, rupture sites, and emergent angiographic findings

\begin{tabular}{cclllr}
\hline Case No. & Age (yrs), Sex & Operation & Rupture Site & Initial Angiogram & Stent Used (mm) \\
\hline 1 & $61, \mathrm{M}$ & TSS & Lt ICA, C4 & Extravasation & $3.5 / 19$ \\
\hline 2 & $31, \mathrm{M}$ & TSS & Lt ICA, C4 & Extravasation & $4 / 12$ \\
\hline 3 & $49, \mathrm{~F}$ & Revision TSS & Rt ICA, C4 & Extravasation & $4 / 12$ \\
\hline 4 & $44, \mathrm{~F}$ & Revision TSS & Rt ICA, C4 & Extravasation & $3.5 / 19$ \\
\hline 5 & $30, \mathrm{~F}$ & TSS & Lt ICA, C4 & Extravasation & $4 / 12$ \\
\hline 6 & $40, \mathrm{~F}$ & TSS & Lt ICA, C4 & Extravasation & $4 / 12,4 / 9$ \\
\hline 7 & $51, \mathrm{~F}$ & TSS & Lt ICA, C5 & Pseudoaneurysm & $4 / 16$ \\
\hline
\end{tabular}

C4 = horizontal cavernous portion; $\mathrm{C} 5$ = ascending cavernous portion. 
TABLE 2. Summary of immediate and follow-up results after covered stent insertion

\begin{tabular}{|c|c|c|c|c|}
\hline Case No. & Treatment Results & Complication & $\begin{array}{l}\text { Follow-Up Imaging } \\
\text { Results (mos) }\end{array}$ & $\begin{array}{l}\text { mRS Score } \\
\quad(\text { mos) }\end{array}$ \\
\hline 1 & $\begin{array}{l}\text { Gap between covered stent \& ICA but w/o leakage; treated w/ coil } \\
\text { insertion, achieving complete occlusiont }\end{array}$ & In-stent thrombosis* & Patent (85) & $0(85)$ \\
\hline 2 & Complete occlusion & None & Patent (48) & $0(56)$ \\
\hline 3 & Complete occlusion & In-stent thrombosis* & Patent (13) & $0(54)$ \\
\hline 4 & Complete occlusion & In-stent thrombosis* & Patent (24) & $0(44)$ \\
\hline 5 & $\begin{array}{l}\text { Initially complete occlusion but leakage on 10-min delay angiogram, } \\
\text { second covered stent inserted, complete occlusion attained }\end{array}$ & None & Patent (38) & $0(40)$ \\
\hline 6 & Complete occlusion & None & Patent (18) & $0(28)$ \\
\hline 7 & Complete occlusion & None & Patent (12) & $0(12)$ \\
\hline
\end{tabular}

* All 3 in-stent thromboses were resolved with intraarterial administration of 5-12 mg of abciximab without any clinical sequelae.

$\dagger$ Coil insertion into the gap between the ICA wall and covered stent 18 hours after completion of the temporarily stopped operation.

follow-up angiogram. Therefore the second covered stent $(4 / 9 \mathrm{~mm})$ was navigated and deployed in a telescopic manner. The immediate (not shown) and 30-minute delayed angiograms showed complete reconstruction of the left ICA without evidence of contrast media leakage (Fig. 2B). The 38-month follow-up angiogram (Fig. 2C) and angiographic CT images (Fig. 2D) showed that the stented segment of the left ICA was patent without in-stent stenosis. The patient had no neurological symptoms at the 30-month clinical follow-up.

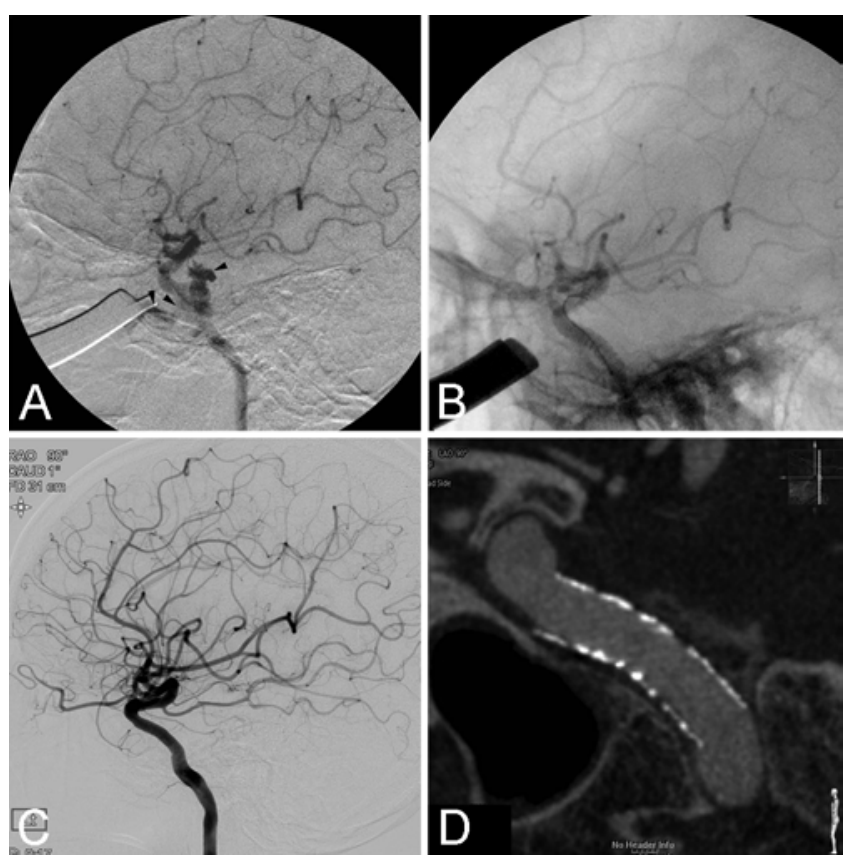

FIG. 2. Case 5. Images obtained in a 30-year-old woman with intraoperative rupture of the left ICA during removal of a pituitary macroadenoma encompassing the cavernous segment of the left ICA. A: Emergency C-arm lateral angiogram in the operating room showing contrast medium extravasation (arrowheads) from the left cavernous ICA into the sphenoid sinus. B: The 30-minute delayed angiogram after insertion of 2 covered stents $(4 / 12 \mathrm{~mm}$ and $4 / 9 \mathrm{~mm})$ showed complete reconstruction of the left ICA without evidence of contrast media leakage or in-stent thrombosis. C and D: The 36-month follow-up angiogram (C) and angiographic CT images (D) showed that the stented left ICA was patent without instent stenosis.

\section{Case 7}

A 51-year-old woman was admitted for removal of a pituitary macroadenoma enveloping the left ICA cavernous segment. A small laceration of the left ICA cavernous segment occurred and was repaired with surgical suture during the operation. The 2-day follow-up angiogram disclosed a small pseudoaneurysm at the descending portion of the left ICA cavernous segment (Fig. 3A). Because postoperative pseudoaneurysms may cause delayed massive epistaxis (as shown in Case 3 ) or progressively enlarge to cause a mass effect, a 4/16-mm covered stent was inserted. A control angiogram after stent placement showed exclusion of the pseudoaneurysm (Fig. 3B). The patient was discharged without any neurological sequelae. The 12-month follow-up angiogram (Fig. 3C) and angiographic CT images (Fig. 3D) showed that the stented left ICA was patent without in-stent stenosis.

\section{Discussion}

Intraoperative rupture of the ICA during TSS is uncommon, but may have catastrophic results if not immediately and properly controlled. If direct surgical repair fails, which is often the case, intraoperative bleeding is controlled by packing with hemostatic agents; however, obtaining hemostasis may be difficult when there is limited access to the ICA due to limited exposure. There are limited numbers of cases investigating cavernous ICA rupture during or after TSS. Raymond et al. ${ }^{16}$ reported 21 cases of arterial injury from 1800 patients who underwent TSS, in which ICA rupture occurred in 17 patients. Profuse intraoperative bleeding from the injured cavernous ICA occurred in 14 patients. Bleeding was controlled by surgical packing in all 14 cases. One patient died of repeat hemorrhage on Day 12. In addition, 1 patient died of epistaxis 2 years later. Two patients experienced immediate infarction, resulting in 1 death. Additionally, 1 patient had delayed infarction 5 months later. Cranial nerve deficits occurred in 6 patients, including 4 who had complete ophthalmoplegia and 3 who had unilateral blindness. Four patients needed additional balloon occlusion of the ICA due to repeat hemorrhage or pseudoaneurysm development.

Except for 1 case that was previously reported and included in this series,,$^{14}$ there have been only 6 cases that 


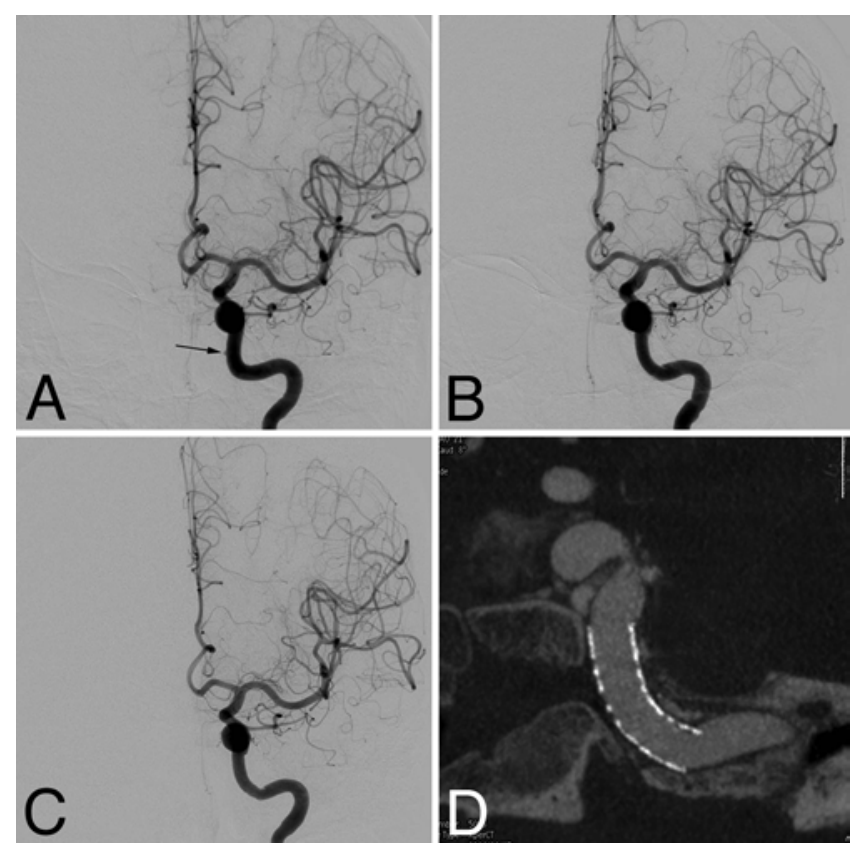

FIG. 3. Case 7. Images obtained in a 51-year-old woman with intraoperative rupture and primary repair of the left ICA cavernous segment during removal of a pituitary macroadenoma. A: The angiogram 2 days after transsphenoidal surgery disclosed a small pseudoaneurysm (arrow) in the descending cavernous segment of the left ICA. B: The control angiogram after a 4/16-mm covered stent was inserted showed exclusion of the pseudoaneurysm. C and D: The 12-month follow-up angiogram (C) and angiographic CT images (D) showed that the stented left ICA was patent without in-stent stenosis.

used a covered stent for reconstruction of a cavernous ICA injury associated with TSS in the literature (Table 3). In these 6 cases, diagnostic angiograms revealed a pseudoaneurysm in 3 cases, a carotid cavernous fistula (CCF) in 2 cases, and active bleeding (extravasation of contrast medium) in only 1 case. Five cases were treated with the same covered stent as was used in this case series, and the other case was treated with a self-expandable covered stent. Immediate posttreatment angiograms showed complete occlusion of angiographic abnormalities in all 6 cases. No symptomatic complications were reported with the exception of 1 transient ischemic attack; however, clinical and imaging follow-up results have not been reported.

In this case series, 6 of 7 patients showed active bleeding on presenting angiograms. Those patients with active bleeding were emergently treated in successive operations $(n=5)$ or just after cardiopulmonary resuscitation $(n=1)$. Although stent-assisted coil placement can be used to reconstruct ICAs in pseudoaneurysms or CCF cases without active bleeding, it cannot be applied to a ruptured ICA with active bleeding. Parent artery trapping is an alternative treatment option; ${ }^{16}$ however, ICA sacrifice requires sufficient cross-collaterals via communicating arteries or otherwise bypass surgery should be completed first. Nonetheless, it is actually impossible to perform the carotid occlusion test in the situation of active bleeding from the ICA. Thus, such emergency situations necessitate prompt decision making to conduct covered stent insertion to keep the ICA patent as well as to immediately control active bleeding. The seventh case showed a pseudoaneurysm on angiography obtained 2 days after primary repair of a lacerated portion of the ICA with suture. Because postoperative pseudoaneurysms may cause delayed massive epistaxis as shown in Case 3 or progressively enlarge to cause a mass effect, ${ }^{1,15,17}$ the patient was also treated with a covered stent.

Navigation of a covered stent can be technically demanding due to its longitudinal stiffness, especially in tortuous ICAs. In this series, however, all attempts at covered stent insertion were successfully completed, although they required simple technical tips in 3 of 7 cases. It is notable that all active bleeding was controlled immediately after covered stent insertion. Although acute in-stent thrombosis occurred in 3 cases, most likely due to lack of antiplatelet premedication, all in-stent thromboses resolved with glycoprotein IIb/IIIa inhibitor administration without any clinical sequelae. These results suggest that covered stents may be a safe and effective treatment option by experienced physicians even in emergency situations with intraoperative or postoperative active bleeding. In addition to the difficult delivery of covered stents, long-term patency is another major concern with covered stent placement in the intracranial ICA.

There are no reports that document follow-up results after covered stent placement for ruptured ICAs during or after TSS. However, follow-up results after covered stent placement for the treatment of traumatic CCF showed good patency without recurrence in 62 (95.4\%) of 65 cases. ${ }^{1,5-7,9,12,18,20}$ In those studies, dual antiplatelet premedication (aspirin $100 \mathrm{mg} /$ day and clopidogrel $75 \mathrm{mg} /$ day) was given to the patients for 3 days. Posttreatment medication depended on each study protocol. Dual antiplatelet therapy was maintained for 3 to 6 months in all studies. Thereafter, antiplatelet medication was stopped in some reports

TABLE 3. Summary of cases with covered stent placement for ICA injury associated with TSS in the literature

\begin{tabular}{lcllll}
\hline \multicolumn{1}{c}{ Authors \& Year } & No. of Cases & \multicolumn{1}{c}{ Finding } & Stent & Immediate Results & Complication \\
\hline Kocer et al., 2002 & 1 & CCF & Jostent & Complete occlusion & None \\
\hline de Souza et al., 2003 & 1 & Pseudoaneurysm & Jostent & Complete occlusion & None \\
\hline Vanninen et al., 2003 & 1 & Pseudoaneurysm & Jostent & Complete occlusion & TIA \\
\hline Leung et al., 2006 & 1 & Pseudoaneurysm & Symbiot $^{*}$ & Complete occlusion & None \\
\hline Kalia et al., 2009 & 1 & CCF & Jostent $^{2}$ & Complete occlusion & None \\
\hline Ghatge \& Modi, 2010 & 1 & Extravasation & Jostent & Complete occlusion & None \\
\hline
\end{tabular}

TIA = transient ischemic attack.

* Self-expanding covered stent. 
but was changed to aspirin monotherapy for the next 3 to 6 months or indefinitely in other reports. . $^{1,5-7,9,12,18,20}$

In the present study, antiplatelet premedication could not be given because stent graft insertion was conducted in the emergency setting of active bleeding. Instead, a loading dose of dual antiplatelet medication (aspirin $100 \mathrm{mg}$ and clopidogrel $300 \mathrm{mg}$ ) was immediately administered via nasogastric tube to all patients. Dual antiplatelet medication was maintained for at least 3-6 months and then changed to aspirin monotherapy indefinitely. No patients suffered clinical symptoms related to covered stent placement during clinical follow-up of a mean duration of 46 months, and all 7 stented ICAs were patent on follow-up vascular imaging at a mean of 34 months (range 12-85 months). In addition, the authors' personal experience of treating 10 cases of CCF with covered stents also showed good patency without any clinical symptoms related to the covered stent (unpublished data). Consistent with previous reports, our results suggest that in terms of its long-term patency, a covered stent placed in the cavernous ICA may be safe.

The small number of cases is a major limitation of this study; however, because ICA rupture requiring emergency covered stent insertion is very uncommon, there are only 6 cases in the literature. Furthermore, follow-up results after covered stent insertion for ruptured cavernous ICAs during TSS have not yet been reported. Therefore, the results of this study may be helpful in making treatment decisions in emergency situations such as ICA rupture during or after TSS.

\section{Conclusions}

Covered stent placement appears to be a safe and effective treatment option for emergency reconstruction of ruptured ICAs during or after TSS.

\section{References}

1. Archondakis E, Pero G, Valvassori L, Boccardi E, Scialfa G: Angiographic follow-up of traumatic carotid cavernous fistulas treated with endovascular stent graft placement. AJNR Am J Neuroradiol 28:342-347, 2007

2. Ciric I, Ragin A, Baumgartner C, Pierce D: Complications of transsphenoidal surgery: results of a national survey, review of the literature, and personal experience. Neurosurgery 40:225-237, 1997

3. de Souza JM, Domingues FS, Espinosa G, Gadelha M: Cavernous carotid artery pseudo-aneurysm treated by stenting in acromegalic patient. Arq Neuropsiquiatr 61 (2B):459-462, 2003

4. Ghatge SB, Modi DB: Treatment of ruptured ICA during transsphenoidal surgery. Two different endovascular strategies in two cases. Interv Neuroradiol 16:31-37, 2010

5. Gomez F, Escobar W, Gomez AM, Gomez JF, Anaya CA: Treatment of carotid cavernous fistulas using covered stents: midterm results in seven patients. AJNR Am J Neuroradiol 28:1762-1768, 2007

6. He XH, Li WT, Peng WJ, Lu JP, Liu Q, Zhao R: Endovascular treatment of posttraumatic carotid-cavernous fistulas and pseudoaneurysms with covered stent. J Neuroimaging 24:287-291, 2014

7. Hoit DA, Schirmer CM, Malek AM: Stent graft treatment of cerebrovascular wall defects: intermediate-term clini- cal and angiographic results. Neurosurgery 62 (5 Suppl 2):ONS380-ONS389, 2008

8. Kalia JS, Niu T, Zaidat OO: The use of a covered stent graft for obliteration of high-flow carotid cavernous fistula presenting with life-threatening epistaxis. J Neurointerv Surg 1:142-145, 2009

9. Kalyanpur TM, Narsinghpura K, Yadav M, Mehta P, Paul $\mathrm{K}$, Cherian M: Covered coronary stent grafts as a treatment option for carotid-cavernous fistulas: our initial experience. Neurol India 59:895-898, 2011

10. Kocer N, Kizilkilic O, Albayram S, Adaletli I, Kantarci F, Islak C: Treatment of iatrogenic internal carotid artery laceration and carotid cavernous fistula with endovascular stentgraft placement. AJNR Am J Neuroradiol 23:442-446, 2002

11. Leung GK, Auyeung KMA, Lui WM, Fan YW: Emergency placement of a self-expandable covered stent for carotid artery injury during trans-sphenoidal surgery. Br J Neurosurg 20:55-57, 2006

12. Li J, Lan ZG, Xie XD, You C, He M: Traumatic carotidcavernous fistulas treated with covered stents: experience of 12 cases. World Neurosurg 73:514-519, 2010

13. Oskouian RJ, Kelly DF, Laws ER Jr: Vascular injury and transsphenoidal surgery. Front Horm Res 34:256-278, 2006

14. Park YS, Jung JY, Ahn JY, Kim DJ, Kim SH: Emergency endovascular stent graft and coil placement for internal carotid artery injury during transsphenoidal surgery. Surg Neurol 72:741-746, 2009

15. Paullus WS Jr, Norwood CW, Morgan HW: False aneurysm of the cavernous carotid artery and progressive external ophthalmoplegia after transsphenoidal hypophysectomy. Case report. J Neurosurg 51:707-709, 1979

16. Raymond J, Hardy J, Czepko R, Roy D: Arterial injuries in transsphenoidal surgery for pituitary adenoma; the role of angiography and endovascular treatment. AJNR Am J Neuroradiol 18:655-665, 1997

17. Reddy K, Lesiuk H, West M, Fewer D: False aneurysm of the cavernous carotid artery: a complication of transsphenoidal surgery. Surg Neurol 33:142-145, 1990

18. Tiewei Q, Ali A, Shaolei G, Feng L, Zhongsong S, Xuesong $\mathrm{L}$, et al: Carotid cavernous fistulas treated by endovascular covered stent grafts with follow-up results. Br J Neurosurg 24:435-440, 2010

19. Vanninen RL, Manninen HI, Rinne J: Intrasellar latrogenic carotid pseudoaneurysm: endovascular treatment with a polytetrafluoroethylene-covered stent. Cardiovasc Intervent Radiol 26:298-301, 2003

20. Wang C, Xie X, You C, Zhang C, Cheng M, He M, et al: Placement of covered stents for the treatment of direct carotid cavernous fistulas. AJNR Am J Neuroradiol 30:1342-1346, 2009

\section{Author Contributions}

Conception and design: BM Kim, DI Kim. Acquisition of data: all authors. Analysis and interpretation of data: BM Kim. Drafting the article: BM Kim, Jeon, DJ Kim. Critically revising the article: BM Kim. Reviewed submitted version of manuscript: BM Kim. Approved the final version of the manuscript on behalf of all authors: BM Kim. Administrative/technical/material support: BM Kim. Study supervision: BM Kim.

\section{Correspondence}

Byung Moon Kim, Division of Interventional Neuroradiology, Department of Radiology, Severance Hospital, Yonsei University College of Medicine, 50 Yonsei-ro, 120-752, Seodaemun-gu, Seoul, South Korea. email: bmoon21@hanmail.net. 